\title{
Effective Lateral Canthal Lengthening with Triangular Rotation Flap
}

\author{
Min Soo Kim \\ Yonsei ENB Plastic Surgery Clinic, Seoul, Korea
}

In Korea, lateral canthoplasty, along with medial epicanthoplasty, has become popular over the past years to widen the horizontal length of the palpebral fissure. However, the effect of the surgery differs greatly depending on the shape and structure of the eyes. If over-widened, complications such as eversion, scarring, and conjunctival exposure may occur. Thus, the author of this study suggests a more effective and safe method for lateral canthal lengthening that causes minimal complications. A total of 236 patients underwent lateral canthoplasty between July 2007 and December 2015. For each patient, a triangular flap 4-5 mm away from the lateral canthus was elevated and rotated 45 degrees laterally while the continuity of the lower eyelid gray line was maintained. A new lateral canthus was created by fixating the rotation flap to the lateral orbital rim with minimal skin trimming and tension-free sutures, preventing relapse and maintaining a triangular shape. In more than 95\% of cases, effective and satisfactory extension was achieved. On average, a $3 \mathrm{~mm}$ extension of the lateral canthus was achieved. There were minor complications such as wound dehiscence, webbing, and scarring, which were easily corrected. The author not only extended the lateral canthus 3-4 $\mathrm{mm}$ laterally but also maintained the continuity of the gray line on the lower lid as a more natural-looking triangular shape, while minimizing complications such as webbing and conjunctival exposure.

Keywords Lateral canthoplasty / Triangular rotation flap / Continuity of the gray line

\author{
Correspondence: Min Soo Kim \\ Yonsei ENB Plastic Surgery Clinic, \\ 842 Nonhyeon-ro, Gangnam-gu, \\ Seoul 06025, Korea \\ Tel: $+82-2-514-2252$ \\ Fax: +82-2-514-2214 \\ E-mail: drbean@naver.com
}

This article was presented at the 830 Taipei Blepharoplasty symposium on Aug 30, 2015 in Taipei, Taiwan.

No potential conflict of interest relevant to this article was reported.

\section{INTRODUCTION}

Double eyelidplasty is the most common type of oculoplastic surgery performed on Asians, but since East Asian eyes typically have relatively short horizontal length, it is often conducted in combination with medial epicanthoplasty and lateral canthoplasty (Fig. 1). Medial epicanthoplasty poses relatively fewer restrictions on the length of extension, since the epicanthus does not contact the eyeball, while an effective lengthening of the lateral canthus is more difficult, since it must stay in contact with the eyeball and plays an important role in maintaining the stability of the lower lid. Recently, lateral canthoplasty has often been used to lower or correct the lateral canthus angle, but complications such as eversion or webbing may occur in the case of eyes with a short horizontal length of $25 \mathrm{~mm}$ or less, or those upslanting by 15 degrees or more (Fig. 2). Various methods like the von Ammon, the Fox, and the Blair methods are used in lateral canthoplasty, but are known to cause frequent side effects or complications [1]. As such, the author would like to report a triangular rotation flap method that includes some parts of the 
gray line of the upper lid as a safer and more effective lateral canthoplasty method.

\section{Fig. 1. Typical East Asian eyes}

Typical East Asian eyes show severe upslanting lateral canthus and short palpebral length.

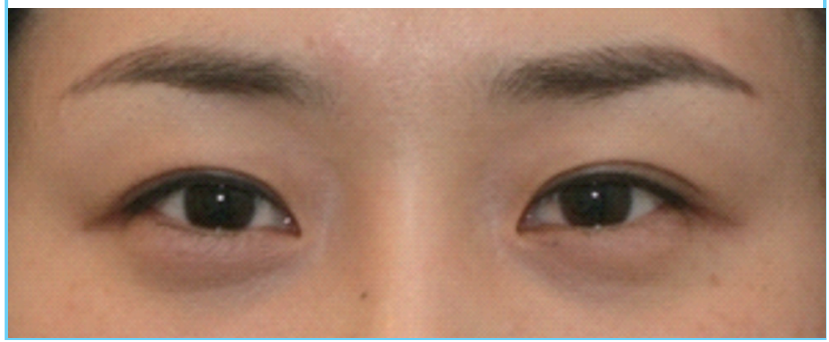

Fig. 2. Frequent complications of lateral canthoplasty

Loss of contact with the eyeball, eversion of the lower lid.

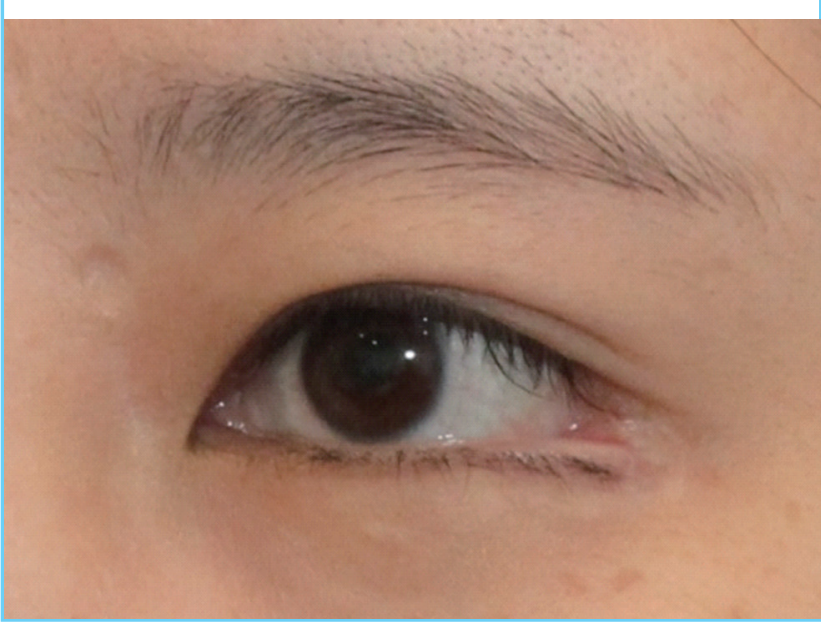

\section{METHODS}

\section{Patients}

Lateral canthoplasty was performed on 236 patients between July 2007 and December 2015. Among the patients, 190 underwent primary lateral canthoplasty, while the remaining 46 underwent re-surgery after failure of the primary operation. The follow-up period was 2 months to 6 years, with an average of 14 months. Patients were evaluated for the presence of ectropion, hypertrophic scar, and the amount of lengthening.

\section{Methods}

The surgical procedure was performed under local anesthesia. Fig. 3 demonstrates the design and the procedure of the opera-

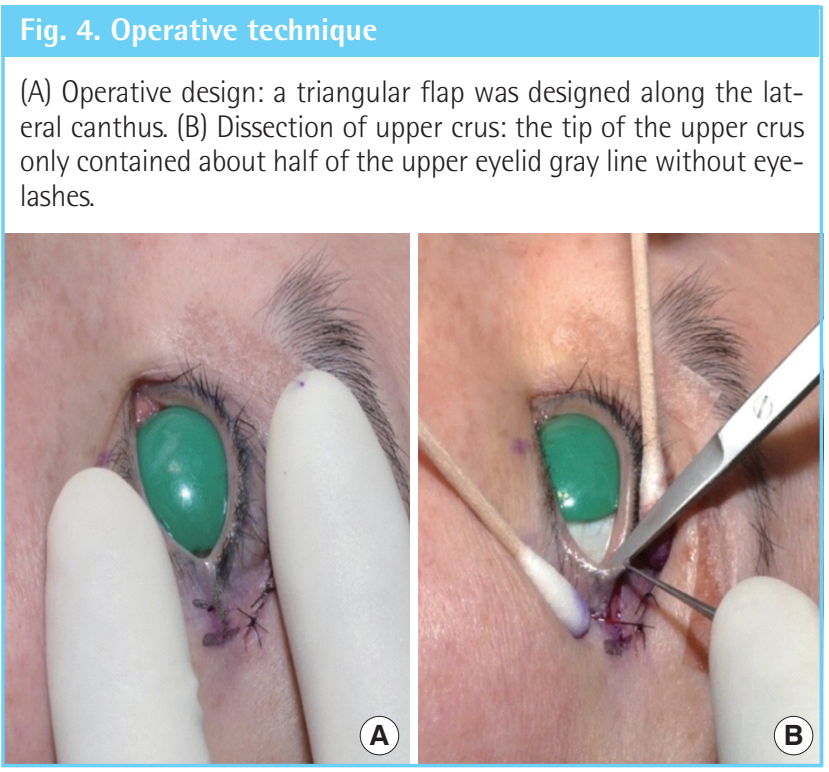

Fig. 3. Schematic drawing of lateral canthoplasty operative technique

(A) Design of the lateral triangular flap: upper crus of flap contains a half portion of the gray line of upper eyelid without eyelashes. (B) Lateral $45^{\circ}$ rotation of the elevated flap: small relaxing incision into palpebral conjunctiva is needed for easy rotation. (C) End of the procedure: the lengthening amount of an extension is about 3-4 $\mathrm{mm}$ from the upper eyelid to the new lateral canthus.
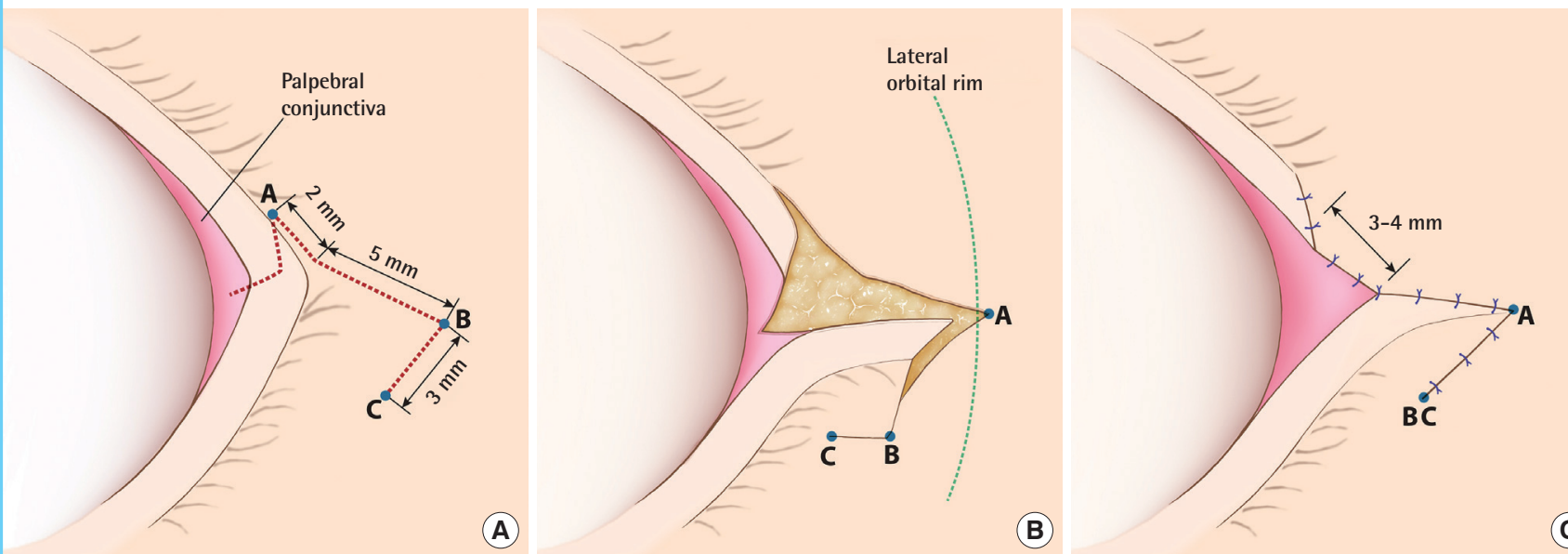
tion. First, the design was drawn around the lateral canthal area in a triangular shape (Fig. 4A). The lateral tip of the triangular flap was about half the length of the distance between the lateral canthus and the lateral orbital rim. The base of the upper eyelid flap contained only about half the portion of the upper eyelid gray line without eyelashes (Fig. 4B). An incision was made with a \#15 scalpel blade along the designed line. The upper eyelid flap was $2 \mathrm{~mm}$ in length and a small relaxing incision was made to the palpebral conjunctiva for easy rotation. The orbicularis oculi muscle around the lateral canthal area was dissected to the periosteum so that the new flap could have enough room to be easily inset and stabilized.

\section{Fig. 5. Operative technique}

(A) Subdermal fixation with \#5-0 PDS to the lateral orbital periosteum. (B) Completion of the sutures.
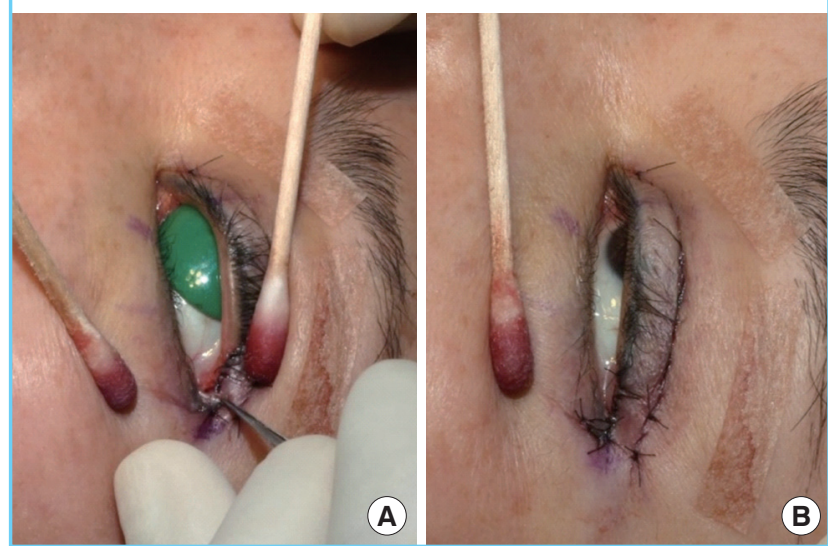

Release of the tarsal strap is necessary for lowering of the lateral canthus angle. After releasing the tarsal strap, the lateral canthus angle could be fixed downward more easily (Fig. 4A). When performing dissection, Eisler's fat pad was preserved as much as possible, though bulging lateral fat was partially removed in some cases [2]. During the process of rotating the triangular flap laterally about 45 degrees, subdermal fixation to the lateral orbital

\section{Fig. 6. Preoperative and postoperative views of a 45-year- old female}

(A) Preoperative view of a 45-year-old female patient. (B) Postoperative 1-week view: note that lateral lengthening of the palpebral fissure is shown.
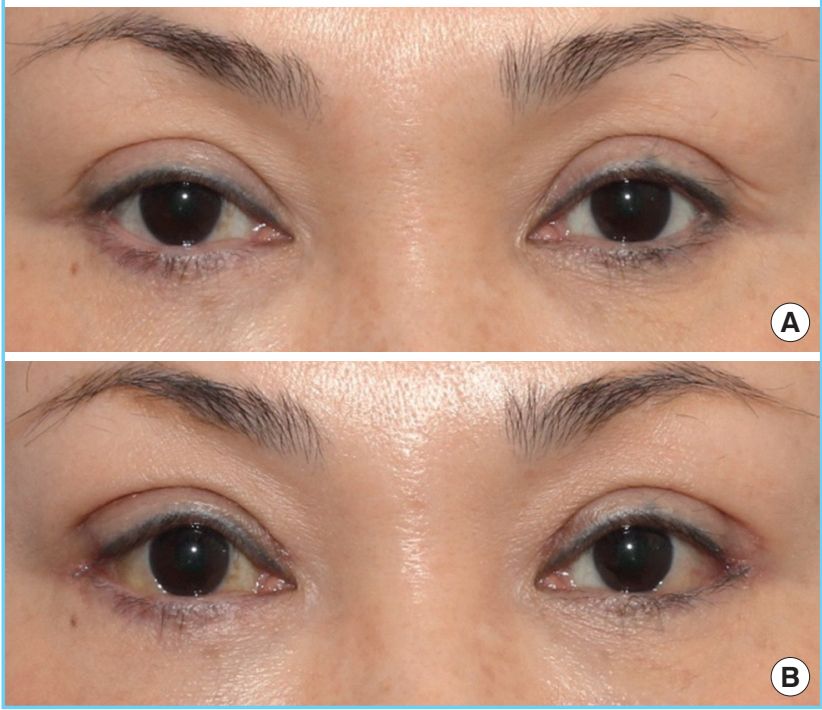

\section{Fig. 7. Preoperative and postoperative views}

(A) Preoperative lateral view of the lateral canthus. (B) Postoperative 5-month view of the newly formed lateral canthus: note that a newly formed lateral canthus should be triangular shaped and maintain contact with the eyeball and show minimal scarring (the double red arrow indicates the lengthened amount).
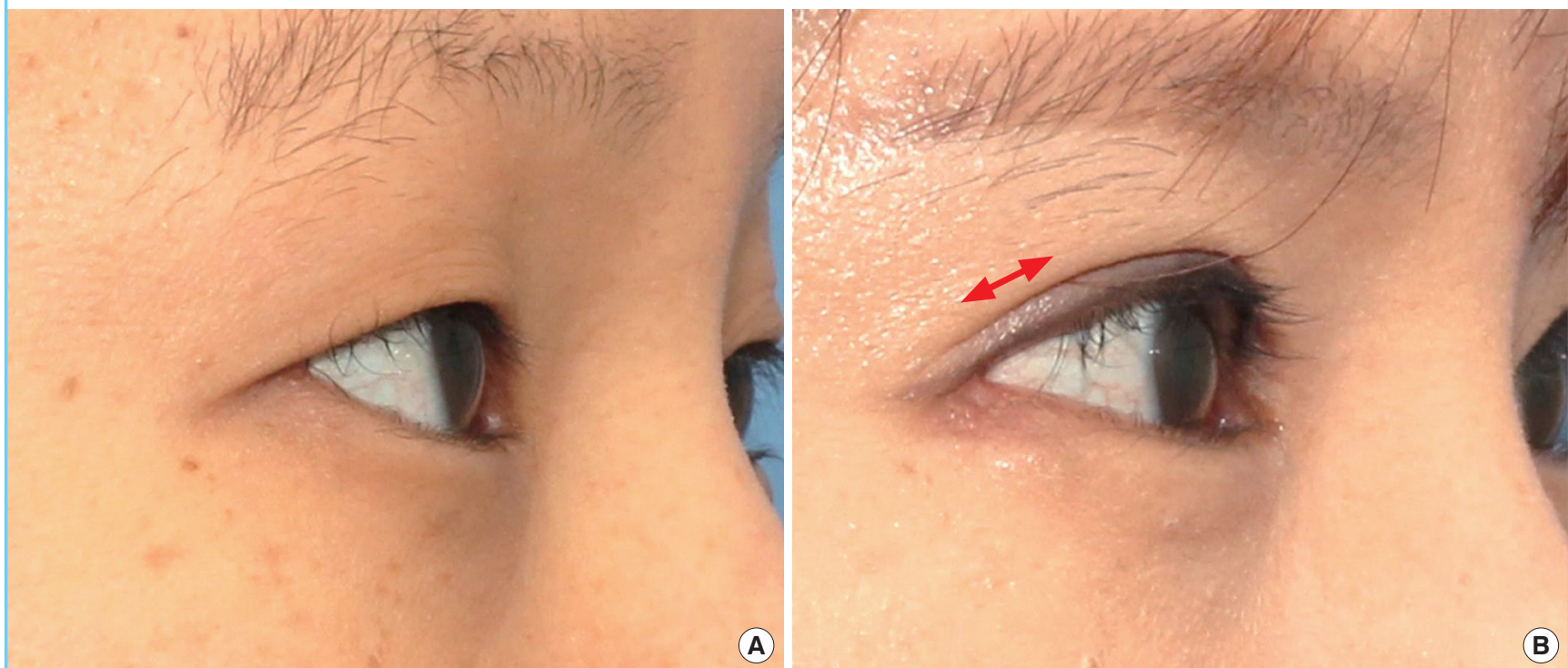


\section{Fig. 8. Preoperative and postoperative views}

(A) Preoperative view. (B) Postoperative 3-month view.

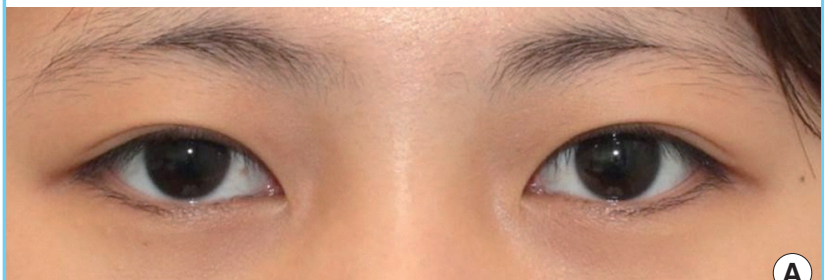

(A)

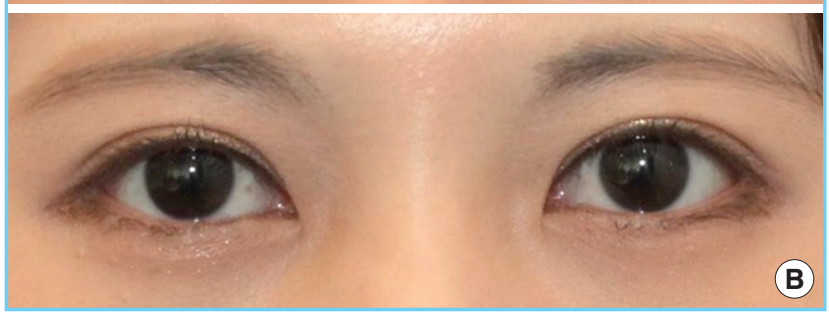

rim was made with a \#5-0 PDS suture. This periosteal fixation suture helps fixate the shape of the newly formed lateral canthus and reduces the tension of the rotation flap, preventing hypertrophic scarring and stabilizing the shape of the canthus. In some cases, subcutaneous tissues were sutured with \#6-0 Vicryl for more secure fixation. Any upper lid defect was sutured directly with \#6-0 black silk sutures. After a new triangular canthus was created, the dog-ear was trimmed and raw surface was sutured (Fig. 4B). All black silk sutures were removed after 1 week (Fig. 5).

\section{RESULTS}

The average amount of lengthening was $3.0 \mathrm{~mm}$ (ranging from $2 \mathrm{~mm}$ to $5.5 \mathrm{~mm}$ ), measured by the length between the original lateral canthus and the newly formed one (Fig. 6). The postoperative shape of the new lateral canthus, presence of scar, lengthening, and the continuity of the gray line were also evaluated. In cases where hypertrophic scar or redness appeared within 3 months after surgery, topical triamcinolone injection or laser ablation for irregularity was performed. A majority of the patients (95\%) were satisfied with the results (Figs. 7-9), but those with narrow palpebral fissure or ptosis, and particularly those with canthal tilt of 10 degrees or more or lateral fornix depth of $2 \mathrm{~mm}$ or less saw relatively little lengthening effect. Two percent of the patients showed webbing deformity, or mucous exposure caused by wound disruption, which was corrected easily.

\section{DISCUSSION}

There have been many lateral canthal lengthening methods reported so far, but they have frequently caused complications such as scarring, webbing, lower lid discontinuity, and loss of

\section{Fig. 9. Preoperative and postoperative views}

(A) Preoperative view. (B) Postoperative 3-months view.
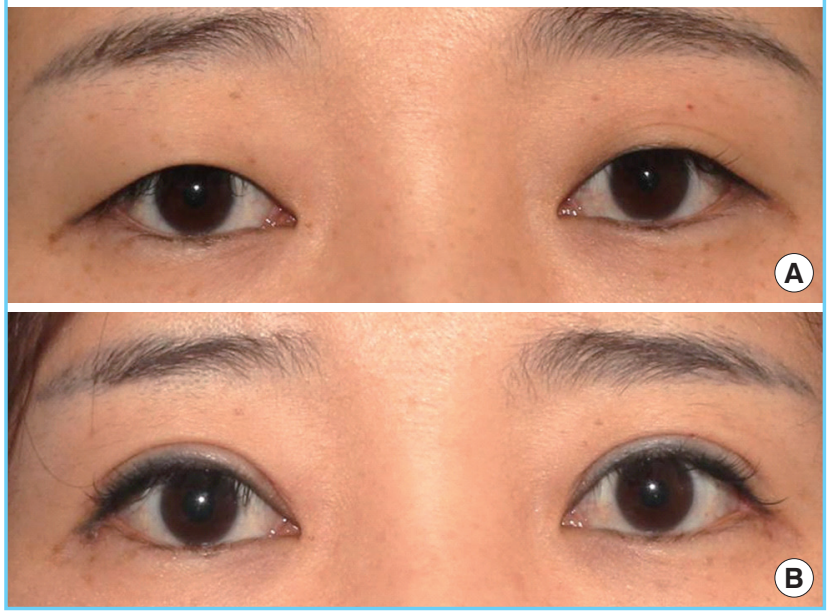

lower lid eyelashes $[1,3,4]$, and the lengthening effect often disappears with time. The author aimed not only at achieving effective extension of the lateral canthus, but also took aesthetic and functional considerations into account. The aesthetic goals of the surgery were: first, making the shape of the newly formed lateral canthus as close to the original triangular look as possible; second, keeping the lateral canthus in contact with the eyeball so that conjunctival prolapse or ectropion should not occur; and third, maintaining the continuity of the lower lid gray line and the new lateral canthus. The functional goals were: first, recovering the dynamic structure that plays the role of sphincter in the lacrimal drainage system [5]; and second, maintaining the tension of the lower lid's curvature $[2,6]$. An effective lateral canthal lengthening not only depends on the distance between the lateral canthus and the lateral orbital rim, but also largely upon the depth of the lateral fornix, which determines the amount of extension. An extension of $2 \mathrm{~mm}$ or greater could be achieved on eyes with fornix depth of $3 \mathrm{~mm}$ or more, while focusing on canthal lowering and deepening would be safer on eyes with fornix depth of $2 \mathrm{~mm}$ or less [1]. The average length between the lateral canthus and the lateral orbital rim is $5.4 \pm 1.5 \mathrm{~mm}$, so an ideal lengthening should be around 3-4 $\mathrm{mm}$ [7]. In order to stabilize the shape and the functions of the newly formed lateral canthus, it should be fixed on the periosteum of the lateral orbital rim with absorbable sutures [8]. This helps reduce the tension of the rotation flap and improve stability. The continuity of the lower lid gray line is critical in the surgery, so attention is required so as not to obliterate the gray line by performing an incision too close to the eyelashes of the lower eyelid when trimming the lower margin of the rotation flap. Too much rotation of the triangular flap or lowering of the lateral canthus could lead to folding or webbing, so the amount of lowering should be 
less than the amount of lengthening in order to maintain the shape and function of the new lateral canthus. The effect of lateral canthal lengthening may not be distinctive from the front view even when the lengthened amount is $3 \mathrm{~mm}$ or longer, because the main effect of lateral canthal lengthening is lateral posterior deepening [1] while giving no significant change to the shape of the lower lid from the front view. Thus, to maximize the front view effect, lower lid lowering must be performed in combination with the lengthening [9].

\section{CONCLUSIONS}

The author's lateral canthal lengthening method involved triangular flap rotation of 45 degrees laterally, which helped achieve aesthetic and functional goals in addition to an average canthal lengthening of $3 \mathrm{~mm}$. This method also helped minimize frequent side effects such as eversion, scarring, webbing, and conjunctival prolapse by maintaining stability through re-anchoring without directly performing canthotomy on the lateral canthus.

\section{REFERENCES}

1. Shin YH, Hwang K. Cosmetic lateral canthoplasty. Aesthetic
Plast Surg 2004;28:317-20.

2. Taban M, Nakra T, Hwang C, et al. Aesthetic lateral canthoplasty. Ophthal Plast Reconstr Surg 2010;26:190-4.

3. Massry GG. Cicatricial canthal webs. Ophthal Plast Reconstr Surg 2011;27:426-30.

4. Nemet AY. The lateral canthus web and its surgical management. J Plast Reconstr Aesthet Surg 2014;67:906-9.

5. Cook T, Goldberg RA, Douglas R, et al. The horizontal dynamic of the medial and lateral canthus. Ophthal Plast Reconstr Surg 2003;19:297-304.

6. Flowers RS, Nassif JM, Rubin PA, et al. A key to canthopexy: the tarsal strap. A fresh cadaveric study. Plast Reconstr Surg 2005; 116:1752-8.

7. Hwang K, Nam YS, Kim DJ, et al. Anatomic study of the lateral palpebral raphe and lateral palpebral ligament. Ann Plast Surg 2009;62:232-6.

8. Hwang K, Kim HJ, Han SH, et al. Where to fix in rejuvenation surgeries?: tensile strength of the periosteum. J Craniofac Surg 2015;26:248-50.

9. Kim MS, Shin YH. Transconjunctival lower eyelid widening using tucking method between lower eyelid retractor and lower tarsal plate. Arch Aesthetic Plast Surg 2012;18:21-5. 